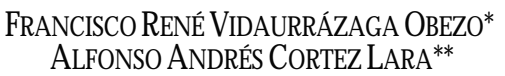

\title{
EL TRATADO DE LIBRE COMERCIO Y LA GANADERÍA BOVINA DE CARNE DE LA REGIÓN FRONTERIZA DE
}

\section{COAHUILA

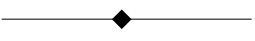 \\ RESUMEN}

El presente trabajo tiene como objetivo analizar la ganadería bovina de carne de la región fronteriza deCoahuila, su importancia en el sector agropecuario dela entidad, así como los efectos del TLC en esta rama pecuaria y sus perspectivas en el corto, mediano y largo plazos.

La ganadería bovina de la región fronteriza de Coahuila, con la operación del TLC atraviesa por una serie de problemas que no le permiten ser competitiva en el corto y mediano plazos. Entre los principales problemas, se puede mencionar que la mayoría de las unidades de producción ganadera presentan un alto grado de endeudamiento con la banca comercial y no tienen solvencia para capitalizar la actividad en el corto plazo, quedando en desventaja con los productores estadounidenses.

\section{A B STR A C T}

This study has the objective to analize the bovine livestock in the N orth region of Coahuila, theimportance on this sector of agricultureand livestock, as well as their effects from NAFTA in the field livestock, with perspectives in a short, medium and long term.

The bovine livestock of the North region of Coahuila is encountering a serie of problems that does not permit to be competitive on a short and medium term, with the operation of NAFTA. According to the principal problems we can mention the bovine industry faces a high debt ratio with financial institutions, and reducing their solvency to meet its capacity on a short term, placing it in desadvantage with United States.

* Investigador de el Colegio de la Frontera Norte, en la Dirección Regional de Piedras Negras, COAHUILA.E-mail:colef@comuni-k.com

** Director de la Dirección Regional de Mexicali de El Colegio de la Frontera norte. E-mail: acortez@telnor.net 


\section{INTRODUCCIÓN}

En los primeros años de la década de los años ochenta, la economía mexicana inició una severa crisis que pronto evidenció ser mucho más profunda que un mero desequilibrio en las finanzas externas del país; se trataba de una crisis de carácter estructural, cuyas causas eran diversas y complejas.

A nte esta situación, el Estado se vio obligado a cambiar el rumbo dela política económica, aplicando una serie demedidas destinadas a lograr la estabilidad económica del país. Entreestas estaban la reducción en la inversión pública, apoyos gubernamentales, eliminación de subsidios así como una apertura comercial unilateral.

Con esta política, el Estado enfrentó al aparato productivo del país a la competencia internacional de una manera muy desigual, que provocó la quiebra financiera y desaparición de la esfera de la producción y del mercado deuna gran cantidad deempresas - sobre todo medianas y pequeñas - que no contaban con los suficientes recursos para enfrentar esta situación de competencia externa.

Entre los sectores de la economía que salieron más perjudicados con la aplicación deestanueva política económica, seencuentrael sector agropecuario, al cual el Estado protegió de una manera parcial en el periodo 1940-1982. Sin embargo, a partir de 1983, retiró gradualmente su intervención en este sector (reducción de la inversión pública agropecuaria, eliminación deapoyos gubernamentales, eliminación de subsidios, precios delos productos, etcétera); esto provocó queel sector agropecuario se descapitalizara de una manera acelerada y redujo grandementesus posibilidades de competencia internacional.

Contrario a la política de desregulación del sector agrícola seguida por M éxico, en losúltimosaños, los paísesindustrializadoshan aplicado una política de sobreprotección de sus actividades agropecuarias. En ese sentido, José Luis Calva (1994) sostiene que en los años ochenta y noventa, los países con sectores agropecuarios vigorosos, como los Estados Unidos, Canadá y la Comunidad Económica Europea principalmente, han reforzado su intervención gubernamental en el campo, inclusive hasta llegar a una guerra de subsidios para hacer 
más competitivos sus productos en el mercado externo; en cambio, en México se produjo una precipitada supresión o reducción de los programas defomento al sector agropecuario.

Los reducidos apoyos que el Estado ha otorgado al sector agropecuario en las décadas de los años ochenta y noventa, obedecea la política macroeconómica dañina, quevol untaria o involuntariamente, discriminaa laagricultura, causando efectosnegativos en su desarrollo. Otro factor que ha incidido en el retraso del sector agropecuario es la falta de visión de los funcionarios gubernamentales que consideran quees más atractivo importar todos los al imentos a un costo muy alto, como lo es el desmantelar el aparato productor dealimentos del país. Sin embargo, se debe tomar en cuenta que la agricultura es un sector estratégico, que contribuye grandemente para evitar que la fuga de divisas sea mayor por concepto de importación de alimentos, a pesar desu escaso desarrollo y apoyos recibidos.

La óptica delos Estados Unidospara formular y aplicar sus políticas en el sector agropecuario se basa en una concepción más allá de simples apoyos aislados; es decir, setieneuna visión integral detodas las actividades que, de manera directa o indirecta, tienen relación con el sector agropecuario, detal manera que los apoyos o subsidios que se otorgan benefician a un sector más amplio que la propia actividad agrícola. En este sentido, Yolanda Trápaga (1992) señala que el sector agrícola estadounidense tiene la particularidad de concebirse como el núcleo que aglutina una importante variedad de actividades económicas, además de otra de orden político y social, y que en su conjunto constituyen el sistema de al imentos y fibras (food and fiber system), que no significa otra cosa que la red productiva y de servicios que depende directamente del renglón de la producción agropecuaria.

Esta concepción de la política reguladora que tienen los Estados Unidos para la agricultura, así como una visión integral de las actividades, les ha permitido tener éxito tanto para el abastecimiento de alimentos para el mercado interno, como para competir a nivel internacional con una gran cantidad deproductos agropecuarios, pues consideran quesi dejaran el sector agrícolaen manos delos particulares, 
los resultados no serían tan halagadores como los que se presentan actualmente, y quelepermiteser el país agrícola másfuertedel mundo.

Ante este panorama tan desal entador para el sector agropecuario, México firmó un Tratado de LibreComercio (TLC) con Estados Unidos y Canadá a finales de 1993. El tratado reconoce las asimetrías existentes entrelostres países, por lo quesefijaron, supuestamente, plazos adecuados para que, aquellos productos agropecuarios donde exista desigual dad en los sistemas de producción, puedan alcanzar el equilibrio o la competitividad detal manera que, al término delos plazos estipulados, se abran totalmente las fronteras. Asimismo, existe un paquete de productos que se liberaron inmediatamente, como es el caso de las plantas de ornato - excepto las rosas-, ganado bovino en pie, carne en canal o en sus diferentes presentaciones, miel de abeja y nuez.

A partir dela firma del TLC, México reconoció la necesidad deapoyar denuevo al sector agropecuario a través desubsi di os y apoyos directos. Desafortunadamente, ya se tienen muchos kilómetros de ventaja por parte de los Estados Unidos y Canadá, por lo que será muy difícil al canzar las condiciones de competitividad en los plazos acordados.

Y es en este marco donde se ubica la ganadería bovina de carne, misma queseliberó inmediatamenteal iniciar operaciones el TLC, bajo el supuesto de quees competitiva; sin embargo, la real idad demuestra lo contrario, pues los directamente afectados (los productores), señal an que la falta de subsidios y apoyos gubernamentales hacen de la ganadería una actividad incosteabley no competitiva.

Chauvet (1991) señala que la inserción de México en el marco mundial de la actividad pecuaria, la ganadería en su conjunto, y particularmente la de bovinos, serían gravemente afectados ante una apertura comercial, ya que puede profundizar la tendencia al aumento de las importaciones, en lugar de incentivar la producción y la productividad.

En este contexto se plantea el presente trabajo, cuyo ámbito de influencia es la región fronteriza deCoahuila (A cuña, Piedras N egras, Jiménez, Allende, Nava, Guerrero, Hidalgo, Villa Unión, Morelos y Zaragoza), región que, por sus características natural es (compuesta de pastizales y matorral es), es propicia para el desarrollo dela ganadería 
bovina de carne de tipo extensivo, principalmente, y además, su producción sedestinaal mercado estadounidenseasí como al interno.

El objetivo del presentetrabajo es anal izar la ganadería dela región fronteriza de Coahuila, su importancia en el sector agropecuario, sus efectos con el Tratado de Libre Comercio, y sus perspectivas en el mediano y largo plazos.

La metodología que se utilizó para la elaboración de este trabajo consistió en el análisis deinformación bibliográfica, estadística, análisis dela encuesta aplicada por la Unión Ganadera Regional deCoahuila, a las asociaciones ganaderas locales del norte de Coahuila, entrevistas a ganaderos de la región, a funcionarios públicos y ex funcionarios vinculados con la ganadería, así como a técnicos de la región.

LA IMPORTANCIA DE LA GANADERÍA BOVINA DE CARNE EN EL SECTOR AGROPECUARIO DE COAHUILA.

\section{ESTRUCTURA PRODUCTIVA GLOBAL.}

Los sectores económicos más dinámi cos dela entidad son el industrial y el terciario, mientras que el sector primario ha disminuido aceleradamente su participación relativa en términos globales en relación con el producto interno bruto (PIB). Estecomportamiento refleja un proceso de industrialización y modernización de la estructura productiva muy semejante al que realiza el país en su conjunto (ver cuadro 1).

En relación con el auge industrial de la entidad, el Plan Estatal de Desarrollo 1994-1999 señala que Coahuila es un estado industrial, con un importantesector exportador, ya que $42 \%$ dela producción total se genera en el sector secundario. Entrelas principales ramas industriales se encuentran la industria automotriz, la industria básica del hierro y del acero, la industria química, la industria de materiales para la construcción, la defabricación y ensamble demaquinaria y equipo, la textil y la del vestido. Estesector absorbe 38\% dela población ocupada. 
CUADRO 1. Producto interno bruto por gran división de actividad económica (1970 a 1990). Estructura porcentual Coahuila.

\begin{tabular}{|l|r|r|r|r|}
\hline \multirow{2}{*}{ Actividad económica } & \multicolumn{5}{|c|}{ A ño } \\
\cline { 2 - 6 } & 1970 & 1980 & 1985 & 1990 \\
\hline Agropecuario, silvicultura y pesca & 9.7 & 6.1 & 6.9 & 6.5 \\
Minería & 5.4 & 5.8 & 5.9 & 5.5 \\
Industria manufacturera & 24.8 & 26.9 & 27.4 & 30.1 \\
Construcción & 5.1 & 6.5 & 5.3 & 4.9 \\
Electricidad & 0.8 & 0.9 & 1.4 & ND \\
Comercio, restaurantes, hoteles, transportes, & 33.1 & 32.5 & 31.9 & 30.9 \\
almacenamiento y comunicaciones & & & & \\
Servicios financieros, seguros, bienes inmuebles, & 21.7 & 21.8 & 21.2 & ND \\
servicios comunales sociales y personales & & & & \\
\hline
\end{tabular}

FueNTE: INEGI, Cuadernos para la Planeación; BANCOMEXT, GerenciaEstatal Coahuila.

En lo que respecta al sector terciario, ésteal igual queel industrial, ha logrado un crecimiento importante, pues desde 1970 aporta poco más de $50 \%$ al producto estatal, y absorbe al rededor de $48 \%$ de la población ocupada en la entidad, según el XI censo depoblación y vivienda 1990.

El sector agropecuario, a pesar desu escasa participación en el PIB estatal, es estratégico para Coahuila por dos razones: la primera, que todavía es asiento de muchas familias campesinas y fuente de trabajo para $12 \%$ de la población ocupada de la entidad; y segundo, genera productos para el consumo y la transformación, así como para la exportación, como el caso del ganado bovino de carne (becerros al destete). 
Una vez analizada demanera general la estructura productiva global de Coahuila, que nos muestra la participación de cada uno de los sectores económicos en el PIB estatal, y además expone un panorama de la evolución económica reciente de la entidad, ahora corresponde analizar el comportamiento interno del sector agropecuario, y particularmente la actividad ganadera.

\section{EL SECTOR AGROPECUARIO Y SU EVOLUCIÓN}

Coahuila cuenta con una superficie de 15157000 ha; de esta superficie, $3.36 \%$ es de uso agrícola, $73 \%$ es para uso ganadero, $20.36 \%$ es de uso forestal maderabley no maderable, y el 3.6\% restantese dedica a otros usos (ver cuadro 2). Los datos anteriores demuestran la importancia que tienela actividad ganadera en la entidad.

CUADRo 2. Uso del suelo en Coahuila.

\begin{tabular}{|c|c|c|c|}
\hline Uso & & Hectáreas & Porciento \\
\hline \multirow[t]{3}{*}{ Agrícola } & Riego & 293718 & \\
\hline & Temporal & 215486 & \\
\hline & Total & 509204 & 3.35 \\
\hline \multirow[t]{3}{*}{ Ganadería } & Extensiva & 9960011 & \\
\hline & Intensiva & 1057510 & \\
\hline & Total & 11017521 & 72.68 \\
\hline \multirow[t]{3}{*}{ Forestal } & Maderable & 185271 & \\
\hline & No Maderable & 2896446 & \\
\hline & Total & 3081717 & 20.33 \\
\hline \multirow[t]{2}{*}{ Otros } & & 548298 & 3.6 \\
\hline & Total & 15157100 & 100.00 \\
\hline
\end{tabular}

FuENTE: Elaboración propia con datos proporcionados por las delegaciones estatal es dela SARH en Coahuila y Comarca Lagunera. 
Entre los principales subsectores que componen el sector agropecuario de Coahuila, se encuentran el agrícola, el pecuario y el forestal. Deéstos, el más dinámico es el subsector pecuario, ya que, en términos devalor dela producción, ocupó 59.9\% para el año de 1980, mientras el subsector agrícola quedó en segundo lugar deimportancia, participando con 39\% para el mismo año; el subsector forestal tuvo una participación poco significativa.

En el periodo 1981-1996, el subsector pecuario tuvo una participación superior a $50 \%$ del valor dela producción total del sector agropecuario y forestal a precios constantes de 1980. Solamente en los años 1981, 1985, 1987 y 1996, la participación fuemenor a 50\%; pero en términos generales muestra una tendencia ascendente. En cambio, el subsector agrícola participó con un porcentaje superior a $40 \%$, pero con una tendencia descendente en dicho periodo. Por último, el subsector forestal mantuvo su participación baja (ver cuadro 3).

Con baseen el anál isisanterior, se deducequeel subsector pecuario dela entidad es el más dinámico. Las principales ramas quecomponen las actividades ganaderas son: la ganadería bovina de carne, la ganadería bovina deleche, la avicultura (carney huevo), la porcicultura, la caprinocultura y la ovinocultura.

En el caso dela ganadería bovina decarne, sobresal en en la entidad, en orden deimportancia, las regiones fronteriza, carbonífera, centro y desierto, las cuales, en conjunto, aportan $80 \%$ de la producción promedio anual, tanto en carne para el abasto del mercado interno, así como becerros al destete para su exportación. Esta actividad es predominantementedetipo extensiva.

Respecto a la ganadería bovino de leche, ésta se concentra en la región de la comarca lagunera del estado, la cual absorbe $75 \%$ del ganado lechero, y aporta 90\% de la producción anual. Esta ganadería cuenta con un nivel tecnológico moderno, por lo que en su mayoría se desarrolla de manera intensiva.

En lo referentea las actividades avícolas, éstas se realizan bajo un esquema detipo moderno, utilizando un nivel detecnol ogía altamente desarrollado, que les permite obtener grandes volúmenes de producción. Al igual que la producción lechera, la avicultura se 
concentra en la región dela comarca lagunera dela entidad, y participa con $70 \%$ de la producción avícola y el otro $30 \%$ se localiza en el resto del estado.

La ganadería caprina y ovina se encuentra distribuida de manera proporcional a lo largo y ancho de Coahuila, a excepción de la región carbonífera, donde el inventario es menor en relación con el resto de las regiones. Esta actividad selleva a cabo de manera extensiva en los agostaderos detipo ejidal, principalmente.

Por último, la actividad porcícola bajo condiciones detipo intensivo, concentra $50 \%$ de la producción en la región de la comarca lagunera y $50 \%$ restante en las regiones fronteriza y sureste.

Delas ramas que componen el subsector ganadero, dos son las más importantes en términos devalor dela producción: la ganadería bovina de carney la ganadería bovina de leche, que en conjunto aportan $60 \%$ promedio anual del valor de la producción (esto en el periodo 19851996, a precios constantes de 1980; ver cuadro 4).

En el cuadro 5 se muestra la participación del ganado bovino de carne en el valor de la producción pecuaria en Coahuila. A parentemente, la ganadería bovina de leche es la más dinámica, a excepción delos años de 1986y 1996, en la quesobresalela ganadería bovina decarne. Sin embargo, es importante aclarar que, en el caso de la ganadería bovina de carne, no se considera el valor de las exportaciones de los becerros al destete, por no tener información disponibleal respecto. En estesentido, el Bancomext (1992) señala que, para el año de 1988, las exportaciones de Coahuila por concepto de ganado bovino ascendieron a catorce millones de dólares; esto lógicamenteincrementó la participación dela ganadería decarneen el valor de la producción pecuaria y, además, rebasaría a la ganadería bovina de leche.

El anál isis anterior demuestra la importancia quetienela ganadería bovina de carne en la economía de Coahuila y en el propio sector agropecuario. La ganadería bovina de carne produce tanto para el mercado interno como para el externo. El becerro al destete es uno de los principales productos de exportación del sector agropecuario de Coahuila. 
CUADRO 3. Valor de la producción agropecuaria y forestal de Coahuila 1980-1996 a precios constantes de 1980. Participación de cada subsector.

\begin{tabular}{|c|c|c|c|c|c|c|c|c|}
\hline Año & Agrícola & Pecuario & Forestal & Agropecuario & Agrícola & Pecuario & Forestal & Agropecuario \\
\hline 1980 & 4490433 & 6889400 & 121332 & 11501165 & 39.0 & 59.9 & 1.1 & 100 \\
\hline 1981 & 4773161 & 2880451 & 66494 & 7720106 & 61.8 & 37.3 & 0.9 & 100 \\
\hline 1984 & 4924216 & 6676812 & 141593 & 11742621 & 42.0 & 56.8 & 1.2 & 100 \\
\hline 1985 & 5268054 & 4206269 & 57108 & 9531431 & 55.3 & 44.1 & 0.6 & 100 \\
\hline 1986 & 4377306 & 4971707 & 58122 & 9407135 & 46.5 & 53.0 & 0.5 & 100 \\
\hline 1987 & 6213373 & 5422996 & 58812 & 11595181 & 53.1 & 46.4 & 0.5 & 100 \\
\hline
\end{tabular}


CUADRO 3. Continuación.

\begin{tabular}{|c|c|c|c|c|c|c|c|c|}
\hline Año & Agrícola & Pecuario & Forestal & Agropecuario & Agrícola & Pecuario & Forestal & Agropecuario \\
\hline 1991 & 4733023 & 6519690 & 79226 & 11331939 & 41.8 & 57.5 & 0.7 & 100 \\
\hline 1992 & 4418913 & 5846998 & 44824 & 10310735 & 42.8 & 56.7 & 0.5 & 100 \\
\hline 1993 & 3186246 & 5999550 & 23945 & 9209741 & 34.6 & 65.1 & 0.3 & 100 \\
\hline 1995 & 3429267 & 5453903 & 37862 & 8921032 & 38.4 & 61.1 & 0.5 & 100 \\
\hline 1996 & 1663104 & 1281821 & 26657 & 2971582 & 56.0 & 43.0 & 1.0 & 100 \\
\hline
\end{tabular}

*Índice de precios al productor $1980=100$

FUENTE. Elaboración propia con información tomada de: Anuario estadístico dela producción agrícola 1980. DGEA-SARH; A gendas de información estadística agropecuariay forestal 1981-1985. DGEA-SARH; anexos técnicos del IV, v y vi Informe deGobierno deCoahuila, dejosédelas Fuentes Rodríguez; A nuarios Estadísticos deCoahuila 1989-199, INEGI; EstadísticasAgropecuarias delas Delegaciones Estatales de SARH-Coahuila y SARH-Comarca Lagunera 1985-1993; Sistema de Cuentas N acionales de México 1980-1991. 
CUADRO 4. Participación total de la ganadería de carne y leche en Coahuila 1985-1996 (en pesos constantes base $1980=100$ ).

\begin{tabular}{|l|c|c|c|}
\hline Año & $\begin{array}{c}\text { Valor dela } \\
\text { producción pecuaria }\end{array}$ & $\begin{array}{c}\text { Valor dela producción } \\
\text { de carney leche } \\
\text { (bovino) }\end{array}$ & $\begin{array}{c}\text { Participación } \\
\%\end{array}$ \\
\hline 1985 & 4206269 & 2327367 & 55.3 \\
1986 & 4971707 & 3172457 & 63.8 \\
1987 & 5422996 & 3369178 & 62.1 \\
1988 & 5976820 & 3746257 & 62.6 \\
1989 & 6203856 & 4218434 & 67.9 \\
1990 & 5611990 & 3542243 & 63.1 \\
1991 & 6519690 & 4101101 & 62.9 \\
1992 & 5846998 & 3419822 & 58.4 \\
1993 & 5999550 & 4191558 & 69.8 \\
1994 & 5719374 & 3340811 & 58.4 \\
1995 & 5453903 & 2967546 & 54.4 \\
1996 & 1281821 & 755288 & 58.9 \\
\hline
\end{tabular}

FUENTE: Elaboración propia con baseen: Estadísticas agropecuarias dela D elegación Estatal de la SARH en el estado de Coahuila, 1985-1990; Estadísticas agropecuarias SARH Región Lagunera, 1988-1992; anexostécnicos del Iv, v y vi informedegobierno de Coahuila, de José de las Fuentes Rodríguez (1985-1987);inEGI. A nuario estadístico del estado de Coahuila (1993, 1994, 1995, 1996, 1997).

Del paquete de productos agropecuarios negociados con el TLC, el ganado bovino decarnees uno de los pocos productos en los cuales se abrieron las fronteras totalmente a partir de la entrada en vigor de dicho tratado; esto supone que no existe asimetría en los sistemas de producción. Sin embargo, el ganado bovino decarneha sido afectado por temporadas considerables a partir dela apertura comercial, ya que mientras los Estados Unidos y Canadá siguen una política de protección, M éxico ha retirado los apoyos y subsidios a esta actividad. 
CUADRO 5. Participación del bovino decarneen el valor dela producción pecuaria de Coahuila 1985-1996 (en pesos constantes base $1980=100$ ).

\begin{tabular}{|l|r|r|r|r|r|}
\hline Año & $\begin{array}{c}\text { Valor dela } \\
\text { producción } \\
\text { pecuaria }\end{array}$ & $\begin{array}{c}\text { Valor dela } \\
\text { producción } \\
\text { bovino decarne }\end{array}$ & $\%$ & $\begin{array}{r}\text { Valor dela } \\
\text { producción } \\
\text { deleche }\end{array}$ & $\%$ \\
\hline 1985 & 4206269 & 1048719 & 24.9 & 1278648 & 30.4 \\
1986 & 4971707 & 2427003 & 48.8 & 745454 & 15.0 \\
1987 & 5422996 & 1429612 & 26.3 & 1939566 & 35.8 \\
1988 & 5976820 & 1824380 & 30.5 & 1921877 & 32.1 \\
1989 & 6203856 & 2025134 & 32.6 & 2193300 & 35.3 \\
1990 & 5611990 & 1795694 & 31.9 & 1746549 & 31.1 \\
1991 & 6519690 & 1976080 & 30.3 & 2125021 & 32.6 \\
1992 & 5846998 & 1594409 & 27.2 & 1825413 & 31.2 \\
1993 & 5999550 & 1541922 & 25.7 & 2649636 & 44.1 \\
1994 & 5719374 & 1221034 & 21.3 & 2119777 & 37.0 \\
1995 & 5453903 & 803937 & 14.7 & 2163609 & 39.7 \\
1996 & 1281821 & 513146 & 40.0 & 242143 & 18.9 \\
\hline
\end{tabular}

FUENTE: Elaboración propia con baseen: Estadísticas agropecuarias dela D elegación Estatal de la SARH en el estado de Coahuila, 1985-1990; Estadísticas agropecuarias SARH R egión Lagunera, 1988-1992; anexos técnicos del Iv, v y vi informedegobierno de Coahuila, de José de las Fuentes Rodríguez (1985-1987);INEGI. A nuario estadístico del estado de Coahuila (1993, 1994, 1995, 1996, 1997).

Marín (1993) sostieneque México eliminó los aranceles y el permiso previo deimportación desde 1988a la carnebovina, y redujo los apoyos y subsidios de una manera desmedida; en cambio, los Estados Unidos y la Comunidad Económica Europea otorgaron el evados subsidios a la ganadería de $34 \%$ y $54 \%$ entre 1988 y 1992, respectivamente. Esta medida generó fuertes importaciones de carne (ver cuadro 6), y tuvo graves consecuencias para la ganadería nacional, tales como problemas financieros, carteras vencidas y comercialización. 
CUADRO 6. Importaciones de carne en canal de ganado bovino en el periodo 1985-1996.

\begin{tabular}{|r|r|}
\hline Año & Toneladas \\
\hline 1985 & 8163 \\
1986 & 689 \\
1987 & 4617 \\
1988 & 28365 \\
1989 & 44242 \\
1990 & 52794 \\
1991 & 131640 \\
1992 & 136963 \\
1993 & 95651 \\
1994 & 119000 \\
1995 & 40103 \\
1996 & 73183 \\
\hline
\end{tabular}

Fuente: A nexo estadístico. V informedegobierno, Carlos Sal inas de Gortari, 1993. Confederación Nacional Ganadera. Informe económico pecuario 1996.

El TLC reconocelas asimetrías entrelos tres países y propone plazos supuestamente adecuados a las características de las distintas ramas del sector agropecuario, para que en el mediano y largo plazos se eliminen esas asimetrías identificadas. La ganadería bovina de carne no está considerada en estos plazos estipulados, por lo que es de gran relevancia el estudio deesta rama del sector agropecuario en cadauna delas grandes regiones del país, y así los resultados orienten con mayor precisión los apoyosinternos y subsidios queel Estado debeotorgar a esta actividad para que pueda subsistir en el mediano y largo plazos.

La frontera norte, es una de las grandes regiones importantes del país, en la que se lleva a cabo la actividad ganadera productora de 
carne, con fuertes nexos al mercado estadounidense. Curiosamente, esta condición beneficia y al mismo tiempo se ve afectada con la operación de un TLC en el corto, mediano y largo plazos. Coahuila es uno de los estados de la frontera norte con el uso del suel o cercano a $80 \%$ para las actividades ganaderas (ver cuadro 2), por lo que se considera preponderantementeganadero.

Bajo este punto de vista, es que se lleva a cabo un estudio de la región ganadera más importante de Coahuila (región fronteriza), que nos permita analizar los efectos y perspectivas dela ganadería bovina de carne en el corto, mediano y largo plazos con la operación de un TLC, cuyas conclusiones finales puedan ser válidas para el resto de las regiones de la entidad y otras zonas de la frontera norte.

ANÁLISISDE LA GANADERÍA BOVINA DE CARNE DE LA REGIÓN FRONTERIZA DE COAHUILA

La región fronteriza de Coahuila comprende diez municipios: Acuña, Piedras N egras, Jiménez, Guerrero, Hidalgo, Zaragoza, Morelos, Nava, Allende y Villa Unión, con una superficie total de 3144324 ha, de las cuales $91.3 \%$ sededica a laganadería extensiva; $3.5 \%$ es para agricultura; $4.2 \%$ se destina a actividades forestales y .9\% es para otros usos (ver cuadro 7). Así, se concluyeque la región es más ganadera queagrícola.

La participación de esta región en el valor de la producción de bovino de carne es de $20 \%$ promedio anual en relación con el valor de la producción total del estado (ver cuadro 8).

El inventario ganadero de la región fluctúa entre 235000 y 240000 cabezas de ganado, que representa $30 \%$ del inventario estatal; sin embargo, en los últimos tres años, este inventario regional se redujo en un 30\%. Los cambios en la política deexportación o la propia apertura comercial, no han influido de manera significativa para ampliar o reducir los inventarios; en cambio, los factores naturales como las sequías, o las heladas, sí han sido determinantes en el aumento o disminución de los inventarios en los años que se presentan estos fenómenos naturales. 
CUADRO 7.Uso del suel o en la región fronteriza de Coahuila.

\begin{tabular}{|c|c|c|c|}
\hline Uso & & Hectáreas & $\%$ \\
\hline \multirow[t]{3}{*}{ Agrícola } & Riego & 59282 & \\
\hline & Temporal & 51746 & \\
\hline & Total & 111028 & 3.53 \\
\hline \multirow[t]{3}{*}{ Ganadería } & Extensiva & & \\
\hline & Intensiva & & \\
\hline & Total & 2872457 & 91.35 \\
\hline \multirow[t]{3}{*}{ Forestal } & Maderable & & \\
\hline & No maderable & & \\
\hline & Total & 132803 & 4.22 \\
\hline \multirow[t]{2}{*}{ Otros } & & 28036 & 0.90 \\
\hline & TOTAL & 3144324 & 100.00 \\
\hline
\end{tabular}

Fuente: Del egación Estatal deAgricultura y Recursos Hidráulicos, D atos básicos de Coahuila 1994.

La ganadería bovina de carne es una actividad que llevan a cabo dos tipos de productores: el sector privado y el sector ejidal. En el caso del primero, éste detenta $80 \%$ de la superficie y obtiene entre $80 \%$ y $90 \%$ de la producción; lo anterior determina que la ganadería bovina de carne sea una actividad principalmente en manos de ganaderos particulares.

De esta manera, el análisis de la problemática de la región lo abordamos a través de las siguientes variables: 
CUADRO 8. Participación dela región fronteriza deCoahuila en el valor dela producción estatal de bovino decarne 1985-1996 (pesos constantes de 1980).

\begin{tabular}{|c|c|c|c|}
\hline Año & $\begin{array}{c}\text { Valor dela producción } \\
\text { del bovino de carne } \\
\text { del estado }\end{array}$ & $\begin{array}{c}\text { Valor dela producción } \\
\text { del bovino de carne } \\
\text { en la región norte } \\
\text { de Coahuila }\end{array}$ & $\%$ \\
\hline 1985 & 1048719 & 273877 & 26.1 \\
1986 & 2427003 & 235260 & 9.6 \\
1987 & 1429612 & 283123 & 19.8 \\
1988 & 1824380 & 298986 & 16.3 \\
1989 & 2025134 & 367550 & 18.1 \\
1990 & 1795694 & 402919 & 22.4 \\
1991 & 1976080 & 463356 & 23.4 \\
1992 & 1594409 & 472056 & 29.6 \\
1993 & 1541922 & 391686 & 25.4 \\
1994 & 1221034 & 287188 & 23.5 \\
1995 & 803937 & 55361 & 7.0 \\
1996 & 513146 & 99182 & 19.3 \\
\hline
\end{tabular}

FuENTE: Elaborado con base en el cuadro 5y Estadísticas pecuarias del distrito de desarrollo rural 001 Acuña-SARH; NEGI, A nuarios estadísticos deC oahuila (1996, 1997).

\section{PRODUCCIÓN}

Los problemas más comunes a los queseenfrenta la ganadería bovina de carne son: escasez de infraestructura, sequías prolongadas, sobrepastoreo, baja calidad genética de los hatos, falta de asistencia técnica, deficiente uso detecnología, deficientesistema demanejo del ganado, bajos rendimientosy, por último, al tos costos de producción.

a) Escasez de infraestructura. Los plazos para recuperar la inversión quese real iza en la creación de infraestructura para el desarrollo dela 
ganadería extensiva son demasiado largos; además, las inversiones son muy altas. Esta situación ha frenado la generación de infraestructura en esta actividad.

Entrelos principales problemas deinfraestructura quese presentan, se encuentra la fal ta de caminos para el transporte de la producción y los insumos, pozos para extracción de agua, aguajes y pastos insuficientes, y cercos (en el caso de los cercos, la mayoría de los ranchos están divididos en dos o cuando mucho en cuatro partes, y esta situación, para realizar un buen aprovechamiento delosranchos, es insuficiente einadecuada).

b) Sequías. Debido a las condiciones geográficas del estado y la región, la condición de aridez de los terrenos es una característica presente en el desarrollo dela ganadería. Las prolongadas sequías en esta área son un riesgo parala producción, lo cual hacequeel inventario ganadero disminuya demanera importantecuando sepresentan estos fenómenos naturales; además, los índices de producción se ven seriamenteafectados, no siendo inmediata su recuperación. No obstante que es una amenaza permanente, la mayoría de los productores no están preparados para sobrellevar esta situación.

c) Sobrepastoreo de los agostaderos. El pastoreo de bovinos se realiza principalmente, en forma extensiva, aprovechando la producción natural del forraje. Sin embargo, el manejo del recurso es inadecuado, lo queha provocado una notable disminución del potencial forrajero.

El cuadro 9 muestra los coeficientes deagostaderos delosmunicipios de la región, encontrándose el coeficiente mínimo en 10.84 ha, y el máximo en 53 ha por unidad animal, con un promedio regional de 16 ha por unidad animal. Lo anterior indica la magnitud del problema en cuanto al manejo de los agostaderos.

d) Baja calidad genética delos hatos. En la región se estima que el $80 \%$ del hato ganadero, es producto de cruzamiento y ganado criollo; de este porcentaje, su mayor parte es ganado híbrido, y el $20 \%$ restante correspondea ganado deal to registro con razacharoláis, cebú y herford.

e) Bajos rendimientos. La falta de aplicación de técnicas adecuadas de manejo, y la baja calidad genética de los hatos, provoca que los rendimientos sean bajos, y esto a su vez, reduce los ingresos de los 
CUADRO 9. Coeficiente de agostadero en los municipios de la región fronteriza deCoahuila (hectáreas/ unidad animal).

\begin{tabular}{|l|c|c|c|}
\hline Municipios & Mínimo & Máximo & Promedio \\
\hline Acuña & 14.46 & 53.00 & 22.03 \\
Jiménez & 10.84 & 18.31 & 13.92 \\
Zaragoza & 11.15 & 28.91 & 18.22 \\
Piedras Negras & 10.84 & 12.99 & 10.98 \\
Morelos & 12.99 & 25.21 & 19.88 \\
Allende & 11.71 & 25.21 & 17.92 \\
Nava & 10.84 & 12.99 & 11.89 \\
Villa Unión & 11.71 & 25.21 & 18.75 \\
Guerrero & 11.71 & 15.82 & 12.53 \\
Hidalgo & 11.71 & 21.90 & 14.59 \\
\hline
\end{tabular}

Unidad animal: Vaca de $450 \mathrm{~kg}$ de peso vivo, gestante y amamantando a su cría (menor de 7 meses) o su equivalente en otros animales/ especie.

Fuente: Revista C oahuila Ganadero,Unión Ganadera Regional, Piedras Negras, Coahuila, junio de 1993.

productores. Esto es válido en el contexto general de la región; sin embargo, existen productores de lo mejor del país con capacidad para competir a nivel internacional, pero no son muy abundantes.

f) A sistencia técnica. La asistencia técnica tradicionalmente era proporcionada por el gobierno federal a través de la Secretaría de A griculturay Recursos Hidraúlicos (SARH), y cubría alrededor de $20 \%$ a $30 \%$ delos productores, principalmente del sector ejidal. Esteapoyo se retiró como producto dela nueva política del Estado hacia el campo.

Actualmente, muy difícil que los ganaderos privados contraten a un técnico pues representa un gasto que no están acostumbrados a realizar, por lo tanto, el manejo del ganado lo llevan a cabo demanera tradicional y hereditaria, degeneración a generación. 
g) D eficienteuso detecnol ogía. Los productores dela región destacan por el nivel tecnológico con el quecuentan en comparación con la media nacional. El índice promedio de pariciones a nivel nacional es de 50\%, mientras que la región está $20 \%$ arriba; es decir, maneja un índice cercano a 70\%. Sin embargo, el índicemínimo aceptablees de $85 \%$, por lo que todavía falta mucho por hacer en lo queal uso de la tecnología serefiere.

Desafortunadamente, aplicar una mejor tecnología (sistema de manejo, sistema de empadre o un empadre controlado) implica una inversión en el corto plazo, cuyo costo es bastantealto para la capacidad económica de la mayoría de las unidades de producción. A unque es importantemencionar queen la región existe un reducido número de ganaderos que emplean un buen nivel de tecnología que les permite ser competitivos tanto a nivel nacional como internacional.

Coahuila y la región tienen cierta ventaja natural en la producción de becerros para el mercado estadounidense, en comparación con el resto delos estados dela frontera norte. Esto se debeal tipo deal imento que consumen en los agostaderos: pastizales y matorrales, pues el matorral permite, por ejemplo, queel animal pueda balancear su dieta de tal manera que se obtenga un mejor peso al destete.

h) Elevados costos de producción. La adquisición dealimentos durante las épocas difíciles del año, los créditos con al tas tasas de interés, y la compra de otrosinsumos a precios al tos en comparación con losprecios bajos a los que se vende el producto, hacen de la ganadería una actividad poco rentable, de tal manera que los productores corren $\mathrm{e}$ riesgo de quedar con carteras vencidas.

\section{COMERCIALIZACIÓN}

En la actividad ganadera bovino de carne, la comercialización del producto (becerros al destete, vacas, vaquillas, torete) se caracteriza por la utilización decanales inadecuados con excesivo intermediarismo y escasa infraestructura y organización de los productores para integrarse a la cría, engorda, matanza y comercialización en los mercados nacional einternacional. 


\section{CRÉDITO}

En la región existe disponibilidad de créditos suficientes, tanto por la banca de desarrollo como la iniciativa privada. Sin embargo, el problema no es la disponibilidad de créditos, sino los requisitos que se tienen quecubrir para tener acceso a éste, entreellos la garantía queexigede 2 a 1; es decir, por cada peso que la banca otorgue, el productor debe tener bienes que garanticen la recuperación del crédito en un $200 \%$. Así, la tasa de interés que se fija, en comparación con las tasas de los Estados Unidos, es demasiado al ta.

Por ejemplo, el crédito a los productores estadounidenses les cuesta $4 \%$ de interés anual; mientras que a los productores mexicanos les aplican una tasa deinterés superior a 20\% anual; no hay comparación, el crédito en M éxico es mucho más caro.

\section{INVERSIONES}

Las actividades ganaderas de la región requieren defuertes inversiones parael desarrollo delainfraestructura productiva y decomercialización, así como la aplicación detecnología moderna. Las unidades de producción se encuentran con un alto grado de endeudamiento (carteras reestructuradas, etcétera), por lo que esta situación no les permite realizar inversiones a corto plazo. Así, únicamente un porcentaje reducido de ganaderos de la región cuenta con la infraestructura adecuada, y lo cual les posi bilita al canzar al tos niveles de producción y productividad.

\section{INVESTIGACIÓN Y DESARROLLO TECN OLÓGICO}

El rezago tecnológico y la falta deasesoría especial izada en la ganadería extensiva de la región, traen como consecuencia bajos niveles de productividad, así como un deficienteaprovechamiento delos recursos naturales.

Para subsanar esta situación, en la región existe un centro de investigaciones agropecuarias que dependedela SAGAR, sin embargo, 
las investigacionesy la generación detecnología sehan orientado más a la agricultura quea la ganadería.

\section{APOYOS GUBERNAM ENTALES}

Los apoyos queel Estado ha otorgado a la actividad ganadera, se han reducido en forma alarmante en los últimos diez años.

En este sentido, los productores consideran que los apoyos para el problema de la sequía y mejoramiento genético no cubren ni siquiera $10 \%$ de las necesidades de la región. Asimismo, mencionan que los programas de apoyo gubernamental, en términos generales, son buenos, pero que la mayor parte de los recursos no llegan al campo, y ésta es la causa de que no haya impactos positivos en las actividades del sector. Por lo tanto, los productores necesitan que el Estado les preste dinero barato y suficiente para capitalizar la actividad.

\section{ORGANIZACIÓN}

Como semenciona al principio del apartado, en el estado deCoahuila existen dos tipos de productores: el productor ejidal y el ganadero particular. En el primer caso, los niveles de organización con los que cuenta son muy deficientes, lo cual obedece, principalmente, a la descapitalización de los productores en los últimos años y a la cancelación decréditos.

En lo que respecta a los ganaderos particulares, aquí encontramos tres niveles de productores: pequeños, medianos y grandes. En la categoría de pequeño productor éstos no están organizados, mientras que los de nivel mediano a grande productor, se encuentran afiliados a las asociaciones ganaderas locales, y éstas a su vez dependen de la Unión Ganadera Regional deCoahuila con influencia en todo el estado -a excepción delos cinco municipios de la Comarca Lagunera.

Es importantemencionar que, para ser integrantede una asociación ganadera local, el ganadero particular debe cubrir una cuota de 8000 pesos por año. Esto definitivamente deja fuera de las asociaciones a una gran cantidad de productores privados. 
La falta de capacitación a los ganaderos es otra de las causas que originan un mal uso y manejo de los recursos naturales, del ganado, y de las técnicas para su mejoramiento genético y nutricional.

En términos generales, ésta es la problemática principal por la que atraviesa la ganadería bovina de carne de la región fronteriza de Coahuila. Otro de los factores que es importante destacar, es que la mayoría de las empresas ganaderas particulares se encuentran con carteras vencidas reestructuradas y con un al to grado de endeudamiento, situación que dificulta la solvencia económica para invertir y capital izar la actividad en el corto y mediano plazos.

De la misma manera, los productores ejidales tienen una mayor desventaja, pues no cuentan con recursos económicos para seguir trabajand o sus unidades de producción, y fuentes financieras como el Banco de Crédito Rural, no son la al ternativa, debido a que la política decrédito actual no les permite obtener los beneficios queestetipo de dependencias puedeofrecer.

LOSEFECTOSDEL TRATADO DE LIBRE COMERCIO EN LA GANADERÍA BOVINA DE LA REGIÓN FRONTERIZA DE COAHUILA

Delas diversas ramas quecomponen el sector agropecuario y forestal, aparentementela ganadería bovina de carnees la menos afectada. Esto motivó al Estado para incluirla en el TLC y liberar su comercio a partir de la entrada en vigor de éste. Otras de las causas fueron la presión que ejerció el gobierno estadounidense, para reducir los al tos inventarios de carne, producto de la disminución del consumo de carnes rojas en el país.

Por otra parte, se dice que la actividad ganadera es muy rentabley quelos productoresqueviven deesta actividad obtienen altos ingresos. Esta versión, más que una realidad, es un mito.

La ganadería bovina de carne de la región fronteriza de Coahuila, al igual que el resto de las regiones del estado y de la frontera norte, está vinculada al mercado estadounidense a través de la exportación 
de becerros al destete. Este hecho, aparentemente, representa al tos ingresos para las empresas ganaderas; sin embargo, la real idad es que la comercialización del producto no se hace a través de las unidades de producción, sino por medio de intermediarios y acopiadores, que en última instancia, son los principal es beneficiados con la exportación a gran escala. Lo mismo sucede en la venta de ganado al mercado nacional.

Con baseen lo ya expuesto, se deducequela producción deganado bovino en la región fronteriza de Coahuila no se destina únicamentea la exportación, sino también al mercado interno. Este planteamiento deriva la siguiente pregunta: ¿Cómo beneficia o afecta el TLC a la ganadería bovina de la región fronteriza de Coahuila? Si se parte del hecho dequees un producto deexportación y el TLC elimina las barreras arancelarias y no arancelarias, esto permiteexportar un volumen mayor de ganado quebeneficiaría a los acopiadores y al país en general, mas no a los productores en particular.

Por otro lado, el volumen de producción de carneque se destina al mercado interno por parte de la región en términos comparativos al volumen exportable, es considerable, y el tratado abre las fronteras para la libreimportación decarnedelosEstados Unidos, lo cual genera una competencia en el mercado interno que afecta a la ganadería mexicana, ya que la carne de importación se ofrece a precios mucho más bajos, gracias a los fuertes apoyos y subsidios que otorga el gobierno de los Estados Unidos a sus productores ganaderos.

Al respecto, García Castells (1994) señala queel TLC podría significar el fracaso dela ganadería en M éxico. Esto con base en queel mercado nacional será invadido por carnedelos EstadosUnidos, como resultado dela desgravación deimpuestos para importar carne. Así por ejemplo, de un 31\% queanteriormentese tenía quepagar para importar carnea México, ahora, con el TLC, el porcentajebajó a cero. Definitivamentelos ganaderos mexicanos no están en condiciones de competir con los productores de Estados Unidos, ya que éstos reciben importantes apoyos y subsidios, y en México no se tiene ese respal do económico.

Regresando al caso regional, algunos funcionarios de la banca sostienen que, del total de ingresos que obtienen las empresas 
ganaderas, $60 \%$ corresponde a la venta de becerros al destete para exportación, y el $40 \%$ restante lo obtienen de la venta de becerros excedentes, vacas de desechos, toretes y vaquillas, los cuales destinan al mercado interno. Es en este último aspecto donde el TLC afecta seriamente a la ganadería regional.

El cuadro 10 muestra un comparativo de los volúmenes de producción de la región enviados para la exportación a través de becerros al destete y al mercado interno, en el periodo 1985-1996.

CUADRO 10. Volumen estimado de exportación de carne (becerros al destete) y producción de carne para el mercado local de la región fronteriza de Coahuila, 1985-1996.

\begin{tabular}{|l|c|c|c|c|c|}
\hline Año & $\begin{array}{c}\text { Volumen } \\
\text { total (t) }\end{array}$ & $\begin{array}{c}\text { Exportación } \\
(\mathrm{t})\end{array}$ & $\%^{*}$ & $\begin{array}{c}\text { Mercado local } \\
(\mathrm{t})\end{array}$ & \%* \\
\hline 1985 & 6188 & 800 & 13.0 & 5388 & 87.0 \\
1986 & 8091 & 2803 & 35.0 & 5288 & 65.0 \\
1987 & 11344 & 5915 & 52.0 & 5429 & 48.0 \\
1988 & 8870 & 3736 & 42.0 & 5134 & 58.0 \\
1989 & 7722 & 1973 & 25.5 & 5749 & 74.5 \\
1990 & 9403 & 2856 & 30.0 & 6548 & 70.0 \\
1991 & 12478 & 5031 & 40.0 & 7448 & 60.0 \\
1992 & 12193 & 4850 & 39.8 & 7343 & 60.2 \\
1993 & 13272 & 6424 & 48.4 & 6849 & 51.6 \\
1994 & 10421 & 2565 & 24.6 & 7856 & 75.4 \\
1995 & 7146 ** & 1143 & 16.4 & 6003 & 84.0 \\
1996 & 3731 & 731 & 19.6 & 3000 & 80.4 \\
\hline
\end{tabular}

*Porcentajerespecto al volumen total.

**Estimado.

FUENTE: Elaboración propia con baseen: Estadísticas dela producción agropecuaria, SARH, Delegación Coahuila, 1985-1993; datos de la Unión Ganadera Regional de Coahuila; datos estadísticos de la tesis de maestría de Laura del Moral, Universidad AutónomaA grariaAntonio Narro, 1993. Datos del FIRA, Delegación Regional Piedras N egras. 
El comportamiento delos mercados refleja queen la mayoría delos años, el volumen mayor sedestinó al mercado interno, presentándose en los años 1987, 1988, 1991 y 1993, cierto equilibrio en los volúmenes destinados a ambos mercados. Lo anterior demuestra quelos ingresos de las empresas ganaderas de la región provienen tanto de la exportación debecerrosal destetecomo dela venta del mercado interno.

Respecto a los porcentajes que representan los ingresos de cada mercado, aparentementelos ingresos másal tosseoriginan por las ventas al mercado interno; sin embargo, los precios del mercado deexportación son mayores. Esto confirmalo quesostienen losfuncionarios dela banca que otorgan crédito a la actividad ganadera, en relación con el origen de losingresos de las empresas ganaderas dela región: 60\% segenera en la exportación, y $40 \%$ por la venta al mercado interno.

En relación con el análisis anterior, el Tratado de Libre Comercio traerá como consecuencia, en el corto plazo, fuertes problemas financieros, carteras vencidas, trabas de comercialización, etcétera.

Como ya semencionó, la región cuenta con tres tipos deproductores en el caso dela pequeña propiedad: los grandes ganaderos, los medianosy los pequeños; indudablementequelos efectos del TLC serán diferentes para cada uno de ellos.

El cuadro 11 muestra la distribución de los pequeños propietarios por tamaño de predios en la región fronteriza de Coahuila. Con base en estecuadro, a los pequeños ganaderos los ubicamos en los predios que oscilan entre 101 a $500 \mathrm{ha}$, y representan $49 \%$ del total; I osmedianos ganaderos se ubican en el rango de 501 a 4000 ha, y representan 33\%; y la categoría de grandes ganaderos la encontramos en el rango de más de 4000 ha y representa $18 \%$.

El efecto del tratado será negativo para los pequeños ganaderos en el mediano plazo, pues los el evados coeficientes de agostadero de la región, y la fal ta de infraestructura, tecnología moderna, y los graves problemas en la comercialización, traerá como consecuencia la desaparición deestetipo deproductores.

Losmedianos productores podrán subsistir en laactividad ganadera, siempre y cuando fortal ezcan su nivel de organización y mejoren sus sistemas de producción. Sin embargo, también corren el riesgo de 
desaparecer a través del proceso decompraventa deranchos, debido a la insolvencia económica para cubrir sus carteras vencidas.

Los grandesganaderos, querepresentan el porcentajemás pequeño del universo deganaderos dela región (18\%), son los más beneficiados con el TLC, pues cuentan con la infraestructura adecuada para la producción, utilizan tecnologías más modernas, obtienen mayor producción y productividad; asimismo, tienen capacidad para exportar directamente y vender en el mercado nacional, además de manejar razas deganado dealto registro.

Otro de los fenómenos quese presenta en la región como producto de la apertura comercial y del TLC, es que alrededor de $20 \%$ de la categoría de grandes ganaderos se han integrado a una parte del proceso de producción decarneestadounidensea través dela engorda de becerros al desteteen praderas artificiales (maquila). Esto significa que ahora al gunas empresas engordadoras de ganado de los Estados Unidos se han instalado en la frontera a través de los ganaderos más fuertes dela región para la engorda de becerros en praderas artificiales (segundo eslabón de la cadena de producción), y posteriormente trasladarlos a los corrales de engorda en su territorio. El objetivo de esta modalidad es disminuir costos de producción, al aprovechar la mano de obra barata.

En el caso de los ejidos dela región, el 20\% se dedica a actividades ganaderas, y controlan $20 \%$ dela superficieganadera dela región. De acuerdo con al gunosfuncionarios públicos vinculados con la ganadería, un ejidatario necesita un mínimo de 80 cabezas (unidad animal) para satisfacer sus necesidades familiares. Con base en este número y considerando un coeficientedeagostadero promedio de 16 ha por unidad animal, un ejidatario requiere de 1280 ha de agostadero para subsistir.

El cuadro 12 muestra la relación de ejidos ganaderos dela región y la superficie promedio por ejidatario; de estos ejidos, solamente tres cubren el mínimo de hectáreas que necesita un productor ejidal para subsistir. A nte este panorama, podemos anticipar que los efectos del TLC en el corto y mediano plazo en el sector ejidal, es la desaparición de la ganadería ejidal dado queseestá presentando un proceso decompra venta de los agostaderos. 
CUADRO 11. Distribución de los pequeños propietarios por tamaño de predio en la región fronteriza de Coahuila.

\begin{tabular}{|l|rrrrrrr|}
\hline & \multicolumn{7}{|c|}{ Número de ejidatarios por rangos (ha) } \\
Municipio & $1-10$ & $11-20$ & $21-50$ & $51-100$ & $101-500$ & $501-400$ & Más de 4000 \\
\hline & & & & & & & \\
Acuña & 32 & 70 & 91 & 13 & 21 & 43 & 99 \\
Allende & 50 & 31 & 42 & 25 & 34 & 9 & 0 \\
Guerrero & 57 & 32 & 49 & 51 & 67 & 64 & 14 \\
Hidalgo & 0 & 0 & 5 & 18 & 32 & 49 & 7 \\
Jiménez & 67 & 32 & 72 & 31 & 14 & 10 & 9 \\
Morelos & 17 & 20 & 45 & 32 & 27 & 13 & 4 \\
Nava & 44 & 100 & 106 & 40 & 132 & 24 & 0 \\
Piedras Negras & 1 & 4 & 17 & 25 & 35 & 8 & 0 \\
Villa Unión & 10 & 13 & 31 & 29 & 41 & 47 & 8 \\
Zaragoza & 66 & 35 & 92 & 76 & 109 & 73 & 51 \\
Total & 334 & 337 & 550 & 340 & 512 & 348 & 192 \\
& & & & & & & \\
\hline
\end{tabular}

FUENTE: Elaboración propiacon información proporcionada por la Delegación Estatal dela Secretaría de la Reforma A graria en Coahuila.

\section{CONCLUSIONES}

La ganadería bovina decarnesecaracterizaba por ser una delas ramas del sector agropecuario más rentables a lo largo y ancho del país. En el caso de la frontera norte, esta actividad ya se encontraba vinculada al proceso productivo estad ounidense desdefinales del siglo pasado, lo que originaba que la mayoría de los productores obtuvieran mejores precios en ese mercado, colocándol os en un estrato social acomodado hasta finales dela década delos años setenta. A principios dela década de los años ochenta, la actividad ganadera empezó a disminuir sus ganancias, entrando gradual mente en un proceso de crisis que se ha manifestado más severamente a partir de finales de los ochenta y 
principios delos noventa; esto como producto dela política deapertura comercial unilateral instrumentada por el Estado a partir deesa época, así como la reducción desmedida de los apoyos y subsidios al sector agropecuario, quehan traído como consecuencia, una descapital ización acel erada de varias ramas del sector agrícola.

CUADRO 12. Relación de ejidos con actividad ganadera en la región fronteriza de Coahuila.

\begin{tabular}{|l|c|c|c|r|}
\hline Nombredel ejido & Municipio & $\begin{array}{c}\text { Superficiede } \\
\text { agostadero (ha) }\end{array}$ & $\begin{array}{r}\text { Número de } \\
\text { ejidatarios }\end{array}$ & $\begin{array}{r}\text { Promedio de } \\
\text { ha/ ejidatario }\end{array}$ \\
\hline Braulio Fernández* & Acuña & 30260 & 196 & 81.6 \\
Dolores* & Acuña & 36506 & 28 & 1303.7 \\
JoseM. Morelos* & Acuña & 44698 & 40 & 1117.4 \\
Mariano Escobedo & Acuña & 71092 & 93 & 764.4 \\
Melchor Múzquiz* & Acuña & 34840 & 53 & 657.3 \\
San Esteban* & Acuña & 19300 & 64 & 301.5 \\
San Esteban deEgipto* Acuña & 8000 & 40 & 200.0 \\
V. Carranza* & Acuña & 37479 & 338 & 110.8 \\
Alfredo V. Bonfil* & Acuña & 9800 & 120 & 81.0 \\
El Tepeyac & Jiménez & 5218 & 31 & 168.0 \\
E. Zapata & Jiménez & 7900 & 52 & 152.0 \\
Nuevo Balcones & Jiménez & 6445 & 60 & 107.0 \\
Palmira & Jiménez & 6372 & 18 & 354.0 \\
San Vicente & Jiménez & 4401 & 27 & 163.0 \\
La Agrícola & Zaragoza & 4450 & 49 & 91.0 \\
NCP Progreso* & Zaragoza & 48769 & 57 & 855.0 \\
NCP Santa Eulalia* & Zaragoza & 170000 & 131 & 1297.0 \\
Las Blancas & Villa Unión & 8640 & 26 & 332.0 \\
Santa Mónica & Guerrero & 3907 & 50 & 78.0 \\
Total & & 558077 & 1473 & \\
\hline & & & & \\
\hline
\end{tabular}

*Ejidos $100 \%$ ganaderos.

FUENTE: Elaborado con información proporcionada por la Delegación Estatal de la SARH en Coahuila. 
Contrario a la política de desregulación del sector agrícola aplicada en M éxico, los países industrializados como los Estados Unidos, Canadá y la Comunidad Económica Europea, han aplicado una política de sobreprotección de sus actividades agropecuarias en los años ochenta y noventa, inclusive hasta llegar a una guerra de subsidios.

Ante estas circunstancias, el Estado mexicano abrió las fronteras a ciertas ramas del sector agropecuario a partir de enero de 1994 con la operación del Tratado de Libre Comercio, como es el caso de la ganadería bovina de carne, que supuestamente se encontraba en condiciones de competir con los Estados Unidos y Canadá.

Los resultados del presente trabajo demostraron lo contrario, pues a pesar dequeen Coahuila la ganadería bovina de carnees una delas ramas más dinámicas del sector agropecuario, así como del subsector pecuario, enfrenta diversos problemas que la sitúan en un nivel de desventaja con respecto a los Estados Unidos, como se demuestra en el apartado de los efectos del TLC en esta rama de la actividad.

En la región de estudio, la actividad ganadera selleva a cabo en su mayoría en condiciones rudimentarias (fal ta deinfraestructura para la producción y comercialización, uso detecnología tradicional), quetrae como resultado bajos rendimientos y pocas posibilidades de competencia en el mercado interno con la libre importación de carne delos Estados Unidos.

La mayoría de las unidades ganaderas particulares de la región se encuentran con carteras vencidas y un al to grado de endeudamiento, situación quedificulta la solvencia económica para invertir y capitalizar la actividad en el corto y mediano plazos.

Losganaderos ejidalestienen una mayor desventajaen comparación con los ganaderos particulares, pues no cuentan con los recursos económicos suficientes para seguir trabajando sus unidades de producción.

Es necesario queel Estado formuley apliqueuna pol ítica defomento pecuario más real ista en el corto, mediano y largo plazos, quepermita el apoyo directo de los productores más débiles, con el fin de evitar la desaparición de los pequeños y medianos ganaderos particulares, así como los de tipo ejidal, ya que se presentaría un proceso gradual de 
compraventa de ranchos en la región para tratar de integrar grandes unidades de producción pecuaria vinculadas al proceso productivo estadounidense.

De esta manera, los grandes productores permanecerán en la actividad ganadera, ya que cuentan con la infraestructura adecuada para la producción y la comercialización; además, utilizan tecnologías modernas, cuentan con capital, y los volúmenes de producción que manejan les permite exportar a gran escala. Asimismo, estos productores podrán integrarsedemanera gradual al proceso productivo estadounidense a través del manejo de praderas artificiales para la engorda de becerros.

\section{BIBLIOGRAFÍA}

Banco Nacional deComercio Exterior (Bancomext) (1992). El perfil estatal de Coahuila. Saltillo, Coahuila. México.

Calva, JoséLuis (1994). "Efectos dela estrategia neoliberal en el campo mexicano". El Financiero, 5 de agosto de 1994, P. 28, Sección Economía, México D.F.

Confederación Nacional Ganadera (CNG) (1996). Informe económico pecuario, México, D. F.

Chauvet, Michelle(1991). “Los desafíos delaganadería anteel A cuerdo de Libre Comercio". En M emoria del XI Seminario de Economía A grícola, Instituto de Investigaciones económicas de la UNAM, México, D.F.

Gobierno del estado deCoahuila (1994). Plan Estatal deD esarrollo 19941999. Saltillo, Coahuila, México.

— (1985). IV Informe deGobierno deJosédelas Fuentes Rodríguez. Anexo Técnico. Saltillo, Coahuila, México.

(1986). V Informe deGobierno de Josédelas Fuentes Rodríguez. Anexo Técnico. Saltillo, Coahuila, México.

— (1987). VI InformedeGobierno deJ osédelas Fuentes Rodríguez. Anexo Técnico. Saltillo, Coahuila, México. 
García Castells, Francisco. 1994. "Amenaza el TLC Acabar con la Ganadería Mexicana”. Periódico El zócalo, 30 de enero de 1994, P. 1/ E. Piedras N egras, Coahuila, México.

Instituto Nacional deEstadística Geografía e Informática (INEGI) (1991). XI Censo de población y vivienda, 1990. Coahuila, Tomo III. Aguascalientes, AGS.

— (1987). Cuadernos de información para la planeación, Coahuila. Aguascalientes, AGS.

(1989). A nuario estadístico del estado de Coahuila. Aguascalientes, AGS.

_ (1990). A nuario estadístico del estado de Coahuila. Aguascalientes, AGS.

_ (1991). A nuario estadístico del estado de Coahuila. Aguascalientes, AGS.

_ (1992). Sistemas de cuentas nacionales de M éxico 1980-1991. Aguascalientes, Ags.

(1993). A nuario estadístico del estado de Coahuila. Aguascalientes, AGS.

_ (1994). A nuario estadístico del estado de Coahuila. Aguascalientes, AGS.

_ (1995). A nuario estadístico del estado de Coahuila. Aguascalientes, AGS.

_ (1996). A nuario estadístico del estado de Coahuila. Aguascalientes, AGS.

(1997). A nuario estadístico del estado de Coahuila. Aguascalientes, AGS.

_ (1994). Sistemas de cuentas nacionales de M éxico 1989-1993. Aguascalientes, Ags.

Marín, Patricia (1993). El Sector Pecuario Nacional Retos y Oportunidades. En Revista La trilla, año III, núm. 25, agostoseptiembre, México, D.F.

Secretaría deA gricultura y Recursos Hidraúlicos(SARH) (1980). A nuario estadístico de la producción agrícola. México, D.F.

(1985). A gen da estadística deinformación agropecuaria y forestal 19811985. México, D.F. 
— (1990). A nuario estadístico dela producción agrícola, Tomo II. M éxico, D.F.

(1991). A nuario estadístico dela producción agrícola, Tomo II. México, D.F. (1992). A nuario estadístico dela producción agrícola, Tomo II. M éxico, D.F. (1990). Estadísticas agropecuarias deC oahuila 1985-1990. Delegación Estatal. Saltillo, Coahuila. ( 1992). Estadísticas agropecuarias de la región de la Comarca 